\title{
On semisimple representations of universal lattices
}

\author{
Daniel K. Shenfeld
}

\begin{abstract}
We study finite-dimensional semisimple complex representations of the universal lattices $\Gamma_{n, k}=\mathrm{SL}_{n}\left(\mathbb{Z}\left[x_{1}, \ldots, x_{k}\right]\right)(n \geq 3)$. One may obtain such a representation by specializing $x_{1}, \ldots, x_{k}$ to some complex values and composing the induced homomorphism $\Gamma_{n, k} \rightarrow \mathrm{SL}_{n}(\mathbb{C})$ with a rational representation of $\mathrm{SL}_{n}(\mathbb{C})$. We show that any semisimple representation coincides, on a subgroup of finite index, with a direct sum of tensor products of representations obtained in this way.
\end{abstract}

Mathematics Subject Classification (2010). 20G35, 53C24, 13F20, 20H05, 20G05, 22E40.

Keywords. Universal lattices, superrigidity, arithmetic groups, arithmetic lattices.

\section{Introduction}

The groups $\operatorname{SL}_{n}(\mathcal{O})(n \geq 3)$, where $\mathcal{O}$ is the ring of integers of a number field share many remarkable properties. For example, they have Kazhdan's property $(T)$, a positive solution to the congruence subgroup problem (CSP), and superrigidity. Y. Shalom noticed an interesting interplay between these groups and the groups $\Gamma_{n, k}=\mathrm{SL}_{n}\left(\mathbb{Z}\left[x_{1}, \ldots, x_{k}\right]\right)$. In [17] he named the groups $\Gamma_{n, k}$ universal lattices, because they can be mapped onto many lattices in $\operatorname{SL}_{n}(F)$ for different locally compact fields $F$, and conjectured that many of the common properties of $\operatorname{SL}_{n}(\mathcal{O})$ are inherited from them. It is unknown, however, whether $\Gamma_{n, k}$ can be embedded as a lattice in a locally compact group (embeddings of $\Gamma_{n, k}$ in $\mathrm{SL}_{n}(\mathbb{C})$ obtained by specializing $x_{1}, \ldots, x_{k}$ to algebraically independent values are not discrete). Therefore, the methods of Margulis theory do not apply naturally to problems regarding $\Gamma_{n, k}$.

A recent remarkable result of Shalom and Vaserstein [18], [21] shows that $\Gamma_{n, k}$ has property $(T)$ for $n \geq 3$ and all $k$, and these are in fact the first known examples of linear groups with $(T)$ that are not arithmetic. In [9], Kassabov and Nikolov show that $\Gamma_{n, k}$ does not have the congruence subgroup property; in fact, its congruence kernel is not even finitely generated (f.g.), although it is central. Apparently, the superrigidity of universal lattices, and their representation theory in general, have not been addressed in the literature. This is the focus of this paper.

Recall the most important feature of representations of $\mathrm{SL}_{n}(\mathcal{O})$, superrigidity: 
Theorem 1.1 ([13], Theorem 7.2). Let $\mathcal{O}$ be the ring of integers of a number field $K, \rho: \mathrm{SL}_{n}(\mathcal{O}) \rightarrow \mathrm{GL}_{r}(F)(n \geq 3)$ a representation, $F$ a field of characteristic 0. Then there exists a rational representation $R_{K / \mathbb{Q}}\left(\mathrm{SL}_{n}\right) \rightarrow \mathrm{GL}_{r}$, defined over $F$, that coincides with $\rho$ on a subgroup of finite index.

Here, $R_{K / \mathbb{Q}}\left(\mathrm{SL}_{n}\right)$ is the restriction of scalars, i.e., an algebraic group $H$ such that $H_{\mathbb{Q}} \cong \mathrm{SL}_{n}(K)$. When $F$ contains the Galois closure of $K$, e.g., $F=\mathbb{C}$, we have $H_{F} \cong \prod_{i=1}^{d} \mathrm{SL}_{n}(F)$ for $d=[K: \mathbb{Q}]([24], 6.1 .3)$. Thus, $\rho$ extends, on a finite index subgroup, to a rational representation of $\prod_{i=1}^{d} \mathrm{SL}_{n}(F)$.

The superrigidity theorem was proved by Margulis, in a more general form. However, when proving the CSP (see 2.1 for definitions) for $\operatorname{SL}_{n}(n \geq 3)$ and $\operatorname{Sp}_{2 n}(n \geq 2)$, Bass, Milnor, and Serre noted that the CSP implies superrigidity for $F=\mathbb{Q}$ ([3], 16.2), a result later extended to the general case in [14] and [13]. Their method does not apply directly to the universal lattices, as these do not enjoy the CSP. However, their congruence kernel is central [9], an important result that we shall refer to multiple times. This allows us to argue along the lines of Bass, Milnor and Serre, with necessary modifications, at least for the case of semisimple representations. It should be noted that this is not the general case, as we show immediately; moreover, unlike $\operatorname{SL}_{n}(\mathcal{O}), \Gamma_{n, k}$ also has infinite representations in characteristic $p>0$, as the reduction mod $p$ shows. Thus, $\Gamma_{n, k}$ has a more intricate representation theory than the arithmetic lattices - ultimately due to the richer ideal structure of $\mathbb{Z}\left[x_{1}, \ldots, x_{k}\right]$ compared with $\mathcal{O}$.

A natural way to obtain representations of $\Gamma_{n, k}$ is by specialization: let $\alpha_{1}$, $\ldots, \alpha_{k} \in \mathbb{C}$. Let $\bar{\alpha}: \mathbb{Z}\left[x_{1}, \ldots, x_{k}\right] \rightarrow \mathbb{C}$ be the ring homomorphism defined by assigning the value $\alpha_{i}$ to $x_{i}$, and, by abuse of notation, we denote the induced homomorphism $\mathrm{SL}_{n}\left(\mathbb{Z}\left[x_{1}, \ldots, x_{k}\right]\right) \rightarrow \mathrm{SL}_{n}(\mathbb{C})$ by $\bar{\alpha}$ as well. Composing $\bar{\alpha}$ with a rational representation $\tau$ of $\mathrm{SL}_{n}(\mathbb{C})$ we obtain a representation of $\Gamma_{n, k}$, and we say that it arises from a specialization, or, by abuse of language, that it is a specialization.

Kazhdan asked whether every representation $\rho: \Gamma_{n, k} \rightarrow \mathrm{GL}_{r}(\mathbb{C})$ arises in this way up to finite index, i.e., whether there exists a finite index subgroup $\Gamma_{0} \leq \Gamma_{n, k}$ so that $\left.\rho\right|_{\Gamma_{0}}$ coincides with a specialization. This is not the case, even for $k=1$, and we present two examples which show why it cannot be true. Our first example shows that $\Gamma_{n, 1}$ has non-semisimple representations, as mentioned above, marking a fundamental difference from the representation theory of arithmetic lattices:

Example 1.2. Let $U \unlhd \mathbb{C}[x]$ be an ideal which can be generated by elements in $\mathbb{Z}[x]$ and is not radical, $\sqrt{U}$ its radical. Then $\mathrm{SL}_{n}(\mathbb{C}[x] / U)$ is a connected linear algebraic group. The natural projection $\mathrm{SL}_{n}(\mathbb{C}[x] / U) \rightarrow \mathrm{SL}_{n}(\mathbb{C}[x] / \sqrt{U})$ is surjective by 2.2, and its kernel consists of unipotent elements, since $U$ contains a power of its radical ([1], 7.14). Hence $\mathrm{SL}_{n}(\mathbb{C}[x] / U)$ is not reductive. By choosing a representation $\bar{\rho}$ of $\mathrm{SL}_{n}(\mathbb{C}[x] / U)$ which is not completely reducible (in particular, it is infinite), we obtain a representation $\rho$ of $\Gamma_{n, 1}$ that is not completely reducible either, since its image is Zariski dense in the image of $\bar{\rho}$, and likewise for any finite index 
subgroup of $\Gamma_{n, 1}$. On the other hand, bearing in mind that all the representations of $\mathrm{SL}_{n}(\mathbb{C})$ are semisimple, it is easily seen that any representation of $\Gamma_{n, 1}$ arising from a specialization is completely reducible.

Even when restricting our interest to semisimple representations of $\Gamma_{n, k}$, there are representations that do not arise from a single specialization:

Example 1.3. Consider the coprime ideals generated by $x$ and $x-1$ in $\mathbb{Z}[x]$. By the Chinese remainder theorem we obtain an isomorphism

$$
\mathbb{Z}[x] / x \cdot(x-1) \cong \mathbb{Z}[x] / x \times \mathbb{Z}[x] /(x-1)=\mathbb{Z} \times \mathbb{Z} .
$$

The natural map

$$
\pi: \mathrm{SL}_{n}(\mathbb{Z}[x]) \rightarrow \mathrm{SL}_{n}(\mathbb{Z}[x] / x \cdot(x-1)) \cong \mathrm{SL}_{n}(\mathbb{Z}) \times \mathrm{SL}_{n}(\mathbb{Z})
$$

is a surjection, as can be seen using the fact that $\mathrm{SL}_{n}(\mathbb{Z})$ is generated by elementary matrices (see 2.2). It follows that $\Gamma_{n, 1}$ has an irreducible $n^{2}$-dimensional representation $\theta$. By the appendix, $\mathrm{SL}_{n}(\mathbb{C})$ has no such representations, hence $\theta$ cannot factor through $\mathrm{SL}_{n}(\mathbb{C})$. This is true for finite index subgroups as well, since the Zariski closure of their image under $\theta$ must be the same as the one of $\Gamma_{n, 1}$, seeing that the latter is Zariski connected.

This example shows that semisimple representations of $\Gamma_{n, k}$ may arise by taking finite tensor products of specializations, i.e., given $\bar{\alpha}_{1}, \ldots, \bar{\alpha}_{m} \in \mathbb{C}^{k}$ and irreducible algebraic representations

$$
\tau_{j}: \mathrm{SL}_{n}(\mathbb{C}) \rightarrow \mathrm{GL}_{r_{j}}(\mathbb{C}) \quad(j=1, \ldots, m),
$$

we obtain an irreducible representation of $\Gamma_{n, k}$, namely $\bigotimes_{j=1}^{m} \tau_{j} \circ \bar{\alpha}_{j}$. We remark that the image of $\tau_{j}$ must actually lie in $\mathrm{SL}_{r_{j}}(\mathbb{C})$ by semisimplicity. This is in fact the general case, bearing a striking similarity to representations of $\operatorname{SL}_{n}(\mathcal{O})$ as discussed above. This is our main theorem:

Theorem 1.4. Let $\rho: \Gamma_{n, k} \rightarrow \mathrm{GL}_{r}(\mathbb{C})(n \geq 3)$ be a semisimple representation. Then there exists a finite index subgroup $\Gamma_{0} \leq \Gamma_{n, k}$ so that $\left.\rho\right|_{\Gamma_{0}}$ decomposes as a direct sum $\bigoplus_{i=1}^{n} \rho_{i}$, where each $\rho_{i}$ is a tensor product of specializations.

It is worth pointing out that in the case $k=0$, our proof reduces to that of Bass, Milnor and Serre for the group $\operatorname{SL}_{n}(\mathbb{Z})$; it also shows superrigidity of $\operatorname{SL}_{n}(\mathcal{O})$, by taking $k=1$ and specializing $x$ to a suitable algebraic number, although here we have to assume complete reducibility.

The paper is organized in the following way. Section 2 contains some preliminary definitions and results. In Section 3, we calculate the profinite completion of 
$\mathbb{Z}\left[x_{1}, \ldots, x_{k}\right]$. For the main theorem, we follow the method for arithmetic groups in [3] with the needed changes in Section 4, reducing the question of semisimple representations of $\Gamma_{n, k}$ to that of the representations of its local factors; these are dealt with in Section 5, and the proof is completed subsequently.

The following notation and conventions are used throughout the text. We put $R_{k}=\mathbb{Z}\left[x_{1}, \ldots, x_{k}\right], \Gamma_{n, k}=\mathrm{SL}_{n}\left(R_{k}\right)$. As we fix $n$ and $k$ throughout the discussion, we use the notation $R=R_{k}$ and $\Gamma=\Gamma_{n, k}$ as well. We denote $k$-tuples by overlined bold letters, e.g., $\overline{\boldsymbol{x}}=\left(x_{1}, \ldots, x_{k}\right)$. Rings are always commutative and unital. Local and semi-local rings are denoted by curly letters, e.g. $\mathcal{A}$ (recall that a semi-local ring is a ring that contains only finitely many maximal ideals). For a ring $A$ and a prime ideal $\mathfrak{a} \leq A, A_{\mathfrak{a}}$ denotes the localization of $A$ at $\mathfrak{a}$, while $A_{\hat{\mathfrak{a}}}$ denotes the completion of $A$ with respect to the a-adic topology, i.e., $\lim _{n} A / \mathfrak{a}^{n}$.

An ideal $U$ of finite index in a ring $A$ is denoted by $U \unlhd_{f} A$; finite index subgroups are denoted similarly. We denote by $\hat{A}$ the profinite completion of $A$, namely, $\hat{A}=\lim _{U \unlhd_{f} A} A / U$. In the same fashion, for a group $G$ we define its profinite completion $\hat{G}=\lim _{N \unlhd_{f} G} G / N$.

Acknowledgements. The author thanks Prof. Alex Lubotzky of The Hebrew University of Jerusalem for his invaluable guidance of this work, including suggesting the problem and numerous ideas. The author also wishes to thank Dr. Tsachik Gelander and Dr. Uzy Hadad of The Hebrew University for insightful discussions and remarks, as well as the reviewers for suggesting various improvements to the manuscript. The results presented in this paper are part of a Master's thesis.

\section{Preliminaries}

2.1. The congruence subgroup property. Let $A$ be a ring, $\Lambda=\mathrm{SL}_{n}(A)$. A principal congruence subgroup in $\Lambda$ is the kernel of the natural map $\Lambda \rightarrow \mathrm{SL}_{n}(A / U)$ for an ideal $U \unlhd A$; we denote it by $\mathrm{SL}_{n}(U)$. If $U \leq_{f} A$ then $\mathrm{SL}_{n}(U) \leq_{f} \Lambda$.

Taking the inverse limit over all finite index principal congruence subgroups, we obtain the congruence completion $\tilde{\Lambda}=\lim \Lambda / \operatorname{SL}_{n}(U)$. There exists a natural map $\pi: \hat{\Lambda} \rightarrow \tilde{\Lambda} ; \operatorname{ker} \pi$ is called the congruence kernel of $\Lambda$, and we say that $\Lambda$ has the congruence subgroup property (CSP) if it is finite.

If $A$ is either local [2] or the ring of integers of a number field [3], then $\mathrm{SL}_{n}(A)$ has the CSP for all $n \geq 3$ but not necessarily for $n=2$ [14]. As mentioned before, the congruence kernel of $\Gamma_{n, k}$ is infinite but central.

In what follows, we denote by $\operatorname{SL}_{n}^{\prime}(A)$ any finite index congruence subgroup of $\mathrm{SL}_{n}(A)$ (not necessarily a proper subgroup).

2.2. Generation by elementary matrices. Let $A$ be a ring. For $1 \leq i \neq j \leq n$, put $E_{i j}(A)=\left\{I+r \cdot e_{i j} \mid r \in A\right\}$, and let $\operatorname{EL}_{n}(A)$ be the subgroup generated by 
all the $E_{i j}(A)$. For large enough $n$ ( $n \geq 2$ is sufficient for all rings considered here), $\mathrm{EL}_{n}(A) \unlhd \mathrm{SL}_{n}(A)$ [12]; by definition, $\mathrm{SL}_{n}(A)$ is generated by elementary matrices iff equality holds. This is the case for all $n \geq 2$ when $A$ is local [2], or when $A$ is the ring of integers of a number field [3]. Suppose that $\mathrm{SL}_{n}(A)$ is generated by elementary matrices; then so is $\mathrm{SL}_{n}(A[x])$ [20]. If, in addition, $A$ is f.g., then $\mathrm{SL}_{n}(A)$ is also f.g. In particular, $\Gamma_{n, k}$ is generated by elementary matrices and f.g. for all $n \geq 2, k \geq 1$.

The following propositions are useful:

Proposition 2.1 ([2], 5.1). Suppose that $\mathcal{A}$ is local, $U \unlhd \mathcal{A}$, and let $\mathrm{EL}_{n}(U)$ be the normal closure of $\mathrm{EL}_{n}(\mathcal{A}) \cap \mathrm{SL}_{n}(U)$ in $\operatorname{SL}_{n}(\mathcal{A})$. Then $\mathrm{EL}_{n}(U)=\operatorname{SL}_{n}(U)$ for any $n \geq 2$.

Proposition 2.2 ([2], 5.2). If $U \unlhd A$ and $A / U$ is semi-local, then the natural homomorphism $\mathrm{SL}_{n}(A) \rightarrow \mathrm{SL}_{n}(A / U)$ is surjective.

2.3. Property ( $\boldsymbol{T})$ and finite abelianizations. Let $G$ be a f.g. group, $S$ a finite set of generators for $G$, and let $\rho$ be a unitary representation of $G$ on a Hilbert space $\mathscr{H}$. A vector $v \in \mathscr{H}$ is called an $(\epsilon, S)$-invariant vector if $\|\rho(s) v-v\|<\epsilon\|v\|$ for all $s \in S$. We say that $G$ has Kazhdan's property $(T)$ if there exists $\epsilon>0$ such that every irreducible unitary representation of $G$ on a Hilbert space $\mathscr{H}$ which has an $(\epsilon, S)$-invariant vector is trivial. This does not depend on the choice of $S$. Property $(T)$ has some important group theoretic consequences; the following is one we shall be using frequently.

Proposition 2.3 ([10]). If $G$ has $(T)$ then $G$ has finite abelianizations, namely, if $H \leq_{f} G$ then $H /[H, H]$ is finite.

$\Gamma_{n, k}$ has $(T)$ and hence finite abelianizations for all $n \geq 3$ [18], [21]. This is not true for $\Gamma_{2, k}$, as it surjects onto $\mathrm{SL}_{2}(\mathbb{Z})$, which contains a finite index free subgroup.

2.4. Regular rings. Let $\mathcal{A}$ be a local ring with maximal ideal $\mathfrak{m}$ and Krull dimension $d$. We say that $\mathcal{A}$ is complete if it is complete for the $\mathfrak{m}$-adic topology. It is always true that the minimal number of generators for $m$ is at least $d$ ([1], ch. 11). A is called regular if $m$ can actually be generated by exactly $d$ elements. If the residue field of $\mathcal{A}$ is of characteristic $p$ and if $p \notin \mathfrak{m}^{2}$, then $\mathcal{A}$ is called unramified.

An extension $K_{f}$ of $\mathbb{Q}_{p}$ of degree $f$ is called unramified if the residue field of $K_{f}$ has exactly $p^{f}$ elements. For every $f \geq 1$ there exists a unique (up to isomorphism) unramified extension $K_{f}$ of $\mathbb{Q}_{p}$ of degree $f$; this is the extension generated over $\mathbb{Q}_{p}$ by a primitive $\left(p^{f}-1\right)$-th root of 1 (see e.g. [22], I.IV). Denote by $\mathcal{O}_{f}$ the ring of integers of $K_{f}$. It is easily seen that $\mathcal{O}_{f}$ is unramified iff $K_{f}$ is unramified.

The following structure theorem will be the cornerstone of the calculations in Section 3: 
Theorem 2.4 ([5], Theorem 15). Let $\mathcal{A}$ be a complete unramified regular local ring. Suppose that $\mathcal{A}$ has characteristic 0 and dimension $d$, with a residue field of characteristic $p$ and degree $f$ over $\mathbb{F}_{p}$. Then $\mathcal{A}$ is isomorphic to a formal power series ring in $d-1$ variables over $\mathcal{O}_{f}$.

A Noetherian ring $A$ is called regular if any localization at a prime ideal is regular. Any ring of integers of a number field is regular, since its localizations are Dedekind domains. If $A$ is a regular ring, then $A[x]$ is also a regular ring ([15], IV.25). It follows that $R_{k}$ is regular.

2.5. Representations of direct products. Let $G_{1}, G_{2}$ be finite groups, $G=G_{1} \times$ $G_{2}, L$ an algebraically closed field, $V$ a finite-dimensional vector space over $L$, and let $\rho: G \rightarrow \operatorname{Aut}_{L}(V)$ be an irreducible representation. It is well known that $\rho$ is isomorphic to a tensor product of irreducible representations of $G_{1}$ and $G_{2}$, i.e., there exist irreducible representations $\left(\rho_{1}, U\right),\left(\rho_{2}, W\right)$ of $G_{1}, G_{2}$, respectively, so that $(\rho, V) \cong\left(\rho_{1} \otimes \rho_{2}, U \otimes W\right)$; the latter representation is given by $\left(g_{1}, g_{2}\right)(u \otimes w)=$ $g_{1} u \otimes g_{2} w$, where $G_{1}$ and $G_{2}$ act by $\rho_{1}$ and $\rho_{2}$, respectively.

The same holds for general groups, but the proofs in the literature seem to treat only some specific cases. We sketch here a proof of this elementary result. Let $\left(\rho_{1}, U\right)$ be an irreducible subrepresentation of $\left.\rho\right|_{G_{1}}$, and let $g \in G_{2}$ be any element. Since $G_{1}$ and $G_{2}$ commute in $G$, we see that $g U$ is also an irreducible subrepresentation of $\left.\rho\right|_{G_{1}}$, and either $g U \cap U=0$ or $g U=U$, in which case, by Schur's lemma, $g$ acts on $U$ as a scalar. Let $U_{1}, \ldots, U_{d}$ be all the irreducible components obtained from $U$ in this way. Then $\bigoplus_{i=1}^{d} U_{i}=V$ since $\rho$ is irreducible. Note that the representations $U_{i}$ are all isomorphic to $U$ (by the action of some $g$ ). We fix isomorphisms $U_{i} \cong U$ and identify all these representations.

Let $W=L^{d}$, and define $\rho_{2}: G_{2} \rightarrow \operatorname{Aut}_{L}(W)$ by the action of $G_{2}$ on the standard basis $\left\{e_{i}\right\}_{i=1}^{d}$ as follows: $g\left(e_{i}\right)=\alpha e_{j}\left(g \in G_{2}, \alpha \in L\right)$ if $g U_{i}=U_{j}$ and the action of $g$ after the identification of $U_{i}$ and $U_{j}$ is multiplication by $\alpha$. It is routine to check that $\rho_{2}$ is well defined, and that $\rho \cong \rho_{1} \otimes \rho_{2}$, from which the irreducibility of $\rho_{2}$ follows as well. It is also straightforward to check that if $\rho$ is continuous, then so are $\rho_{1}$ and $\rho_{2}$.

Remark 2.5. Let $G=\prod_{i=1}^{\infty} G_{i}, \rho$ a finite-dimensional irreducible representation of $G$. We may find by induction irreducible representations $\rho_{t}, \rho_{t}^{\prime}$ of $G_{t}, \prod_{i=t+1}^{\infty} G_{i}$, respectively, so that $\rho=\rho_{1} \otimes \cdots \otimes \rho_{t} \otimes \rho_{t}^{\prime}$. Since $\rho$ is finite dimensional, $\operatorname{dim} \rho_{t}^{\prime}=1$ for all but finitely many $t$.

\section{The profinite completion of $\mathbb{Z}\left[x_{1}, \ldots, x_{k}\right]$}

From now on we fix $n$ and $k$ and put $R=R_{k}, \Gamma=\Gamma_{n, k}$. In this section we calculate the profinite completion of $R$. 
Proposition 3.1. Let $A$ be a Noetherian ring. Then $\hat{A}=\prod_{\mathfrak{m} \unlhd A} A_{\hat{\mathfrak{m}}}$, where $\mathfrak{m}$ ranges over all finite index maximal ideals.

Proof. Let $\Phi: \hat{A} \rightarrow \prod_{\mathfrak{m} \unlhd A} A_{\widehat{\mathfrak{m}}}$ be the natural map, and let $U \unlhd_{f} A$ be a finite index ideal, with primary decomposition $U=\bigcap \mathfrak{q}_{i}$ where $\mathfrak{m}_{i}=\sqrt{\mathfrak{q}_{i}}$ are distinct and necessarily maximal, being prime ideals of finite index. For some $t_{i}$, we have $\mathfrak{m}_{i}^{t_{i}} \subseteq \mathfrak{q}_{i}$, since any ideal in $A$ contains a power of its radical. Note that for $i \neq j, \mathfrak{m}_{i}^{t_{i}}$ and $\mathfrak{m}_{j}^{t_{j}}$ are coprime, hence by the Chinese remainder theorem $\bigcap \mathfrak{m}_{i}^{t_{i}}=\prod \mathfrak{m}_{i}^{t_{i}} \subseteq U$, implying injectivity of $\Phi$. Now for $i \neq j, \mathfrak{q}_{i}$ and $\mathfrak{q}_{j}$ are coprime, so again by the Chinese remainder theorem $A / U \cong \prod A / \mathfrak{q}_{i} \subseteq \prod A / \mathrm{m}_{i}^{t_{i}}$, from which surjectivity of $\Phi$ follows.

Note that $R$, being a f.g. ring, does not surject onto any infinite field, hence any maximal ideal in $R$ has finite index.

Proposition 3.2. Let $\mathfrak{m} \unlhd R$ be a maximal ideal. Suppose that $R / \mathfrak{m}$ has $p^{f}$ elements and let $\mathcal{O}_{p, f}$ be the ring of integers of the unramified extension of $\mathbb{Q}_{p}$ of degree $f$. Then $R_{\widehat{\mathfrak{m}}} \cong \mathcal{O}_{p, f}\left[\left[T_{1}, \ldots, T_{k}\right]\right]$.

Proof. $R_{\widehat{\mathrm{m}}}$ is a complete local ring and $\operatorname{dim} R_{\widehat{\mathrm{m}}}=\operatorname{dim} R=k+1$ (cf. [1], 11.19). It is also regular, since $R$ is regular. Since $m$ is a prime ideal of finite index in $R$, we have $p \in \mathfrak{m}$ for some prime $p \in \mathbb{Z}$. We claim that $R_{\widehat{\mathfrak{m}}}$ is unramified, i.e., $p \notin \mathfrak{m}^{2}$; by ([5], Theorem 2), it is enough to show that $R_{\mathfrak{m}}$ is unramified. There is a natural isomorphism $R_{\mathfrak{m}} / p \cong \mathbb{F}_{p}[\overline{\boldsymbol{x}}]_{\overline{\mathfrak{m}}}$ where $\overline{\mathfrak{m}}$ is the image of $\mathfrak{m}$ under the projection modulo $p$. The quotient of a regular local ring by an ideal $U$ is regular iff $U$ can be generated by elements that are linearly independent over $\mathfrak{m} / \mathfrak{m}^{2}$ ([23], 11.26). As the ring on the right is regular, it follows that $p \notin \mathfrak{m}^{2}$, as desired. Note in particular that $\mathfrak{m}$ can be generated by $p$ together with $k$ additional elements, whose image in $\mathbb{F}_{p}[\overline{\boldsymbol{x}}]$ generate $\overline{\mathrm{m}}$. The proposition now follows from 2.4 .

We denote $\mathcal{R}_{p, f}=\mathcal{O}_{p, f}\left[\left[T_{1}, \ldots, T_{k}\right]\right]$. This is a pro- $p$ ring, whose topology clearly coincides with the $\mathfrak{m}$-adic topology on $R_{\widehat{\mathfrak{m}}}$. Note that the indeterminates $\overline{\boldsymbol{T}}$ are $n o t$ the image of the indeterminates $\bar{x}$ in $R$. In fact, from the proof of 2.4 it follows that the isomorphism can be chosen to map the generators of $\mathfrak{m}$ other than $p$ to $\overline{\boldsymbol{T}}$. Putting 3.1 and 3.2 together we obtain:

\section{Proposition 3.3.}

$$
\hat{R} \cong \prod_{p} \prod_{\overline{\mathfrak{m}}} \mathcal{O}_{p, f(\overline{\mathrm{m}})}\left[\left[T_{1}, \ldots T_{k}\right]\right],
$$

where $p$ goes over all primes in $\mathbb{Z}, \overline{\mathfrak{m}}$ goes over all maximal ideals in $\mathbb{F}_{p}[\overline{\boldsymbol{x}}]$, and $f(\overline{\mathrm{m}})$ is the degree of $\mathbb{F}_{p}[\overline{\boldsymbol{x}}] / \overline{\mathrm{m}}$ over $\mathbb{F}_{p}$.

Remark 3.4. The isomorphism in 2.4 is neither unique nor natural, thus neither is the isomorphism in 3.3 . Throughout the discussion, we fix an isomorphism and identify $\hat{R}$ with the above product. 


\section{The Bass-Milnor-Serre argument}

We follow the argument of Bass, Milnor and Serre [3] for arithmetic lattices, which uses finiteness of the congruence kernel to associate a given representation with a map of analytic pro- $p$ groups. In our case the congruence kernel of $\Gamma$ is infinite; we overcome this obstacle by restricting our interest to semisimple representations and using the centrality of the congruence kernel. First, we may shift our focus to $p$-adic representations of $\Gamma$ :

Lemma 4.1. Let $\rho: \Gamma \rightarrow \mathrm{GL}_{r}(\mathbb{C})$ be a representation. Then there exists a prime $p$ and an isomorphism of abstract fields $\Phi: \overline{\mathbb{Q}}_{p} \cong \mathbb{C}$, where $\overline{\mathbb{Q}}_{p}$ is an algebraic closure of $\mathbb{Q}_{p}$, so that under the induced isomorphism on $\mathrm{GL}$, the image of $\rho$ is contained in $\mathrm{GL}_{r}\left(\mathbb{Z}_{p}\right)$.

Proof. $\Gamma$ is f.g. (Section 2.2), hence the entries in the image of $\rho$ generate a f.g. domain $D$, which can be embedded in $\mathbb{Z}_{p}$ for infinitely many primes $p$ ([4], ch. 5). Fixing such a prime $p$ we have $\rho: \Gamma \rightarrow \mathrm{GL}_{r}\left(\mathbb{Z}_{p}\right)$. By elementary field theory considerations, the embedding $D \hookrightarrow \mathbb{Z}_{p}$ can be extended by an embedding $\mathbb{Z}_{p} \hookrightarrow \overline{\mathbb{Q}}_{p} \cong \mathbb{C}$ fixing $D$, and the lemma follows.

From now on we treat irreducible $p$-adic representations of $\Gamma$, returning to the complex case only in the proof of the main theorem. We endow $\overline{\mathbb{Q}}_{p}$ with the topology induced by the unique extension of the $p$-adic norm ([11], III.3).

Proposition 4.2. Let $\rho: \Gamma \rightarrow \mathrm{GL}_{r}\left(\mathbb{Z}_{p}\right)$ be an irreducible representation. Then there exists a finite index subgroup $\Gamma_{0} \leq \Gamma$, so that $\left.\rho\right|_{\Gamma_{0}}$ decomposes as a finite direct sum $\bigoplus \rho_{i}$, where

$$
\rho_{i}: \Gamma_{0} \stackrel{\iota}{\rightarrow} \prod_{j=1}^{t(i)} \mathrm{SL}_{n}^{\prime}\left(\mathcal{R}_{p, f_{j}}\right) \stackrel{\nu}{\rightarrow} \mathrm{GL}_{r}\left(\overline{\mathbb{Q}}_{p}\right)
$$

is an irreducible representation; 1 is the natural embedding, while $v$ is given by a tensor product of continuous irreducible representations.

Proof. Since $\rho$ is irreducible, the Zariski closure $\overline{\rho(\Gamma)}$ of $\rho(\Gamma)$, is reductive, hence its commutator is semisimple. Since $\Gamma$ has finite abelianizations (see 2.3), we may take a finite index subgroup $\Gamma_{0} \leq \Gamma$ so that $\overline{\rho\left(\Gamma_{0}\right)}$ is connected and semisimple. Furthermore, $\mathrm{GL}_{n}\left(\mathbb{Z}_{p}\right)$ is an analytic pro- $p$ group, so it containts an open pro- $p$ torsion-free subgroup ([6], ch. 4). Taking a suitable finite index subgroup again, we may also assume that $\rho\left(\Gamma_{0}\right)$ is torsion-free. It follows that $Z\left(\rho\left(\Gamma_{0}\right)\right)$ is trivial, since its Zariski closure is contained in $Z\left(\overline{\rho\left(\Gamma_{0}\right)}\right)$ which is finite by semisimplicity.

Consider the natural map $\pi: \hat{\Gamma} \rightarrow \widetilde{\Gamma}$ from the profinite completion of $\Gamma$ to its congruence completion. We contend that $\widetilde{\Gamma}=\mathrm{SL}_{n}(\hat{R})$ and that $\pi$ is surjective. Note first that for $U \unlhd_{f} R, \mathrm{SL}_{n}(R) / \mathrm{SL}_{n}(U) \cong \mathrm{SL}_{n}(R / U)$ by Proposition 2.2. Now we 
have natural maps

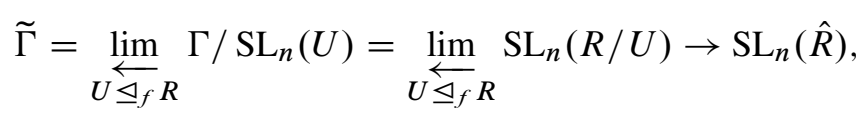

where the last map is easily seen to be injective. It is also surjective, as $\hat{R}$ is local, so $\mathrm{SL}_{n}(\hat{R})$ is generated by elementary matrices, which are clearly in the image of $\widetilde{\Gamma}$. The image under $\pi$ of the elementary matrices of $\Gamma$ (embedded in $\hat{\Gamma}$ ) is dense in the elementary matrices in $\widetilde{\Gamma}$, hence $\pi(\hat{\Gamma})$ is dense in $\widetilde{\Gamma}$; as $\hat{\Gamma}$ is compact and $\pi$ is continuous, it is onto.

Putting $\widetilde{\Gamma}_{0}=\pi\left(\hat{\Gamma}_{0}\right)$ we obtain a diagram

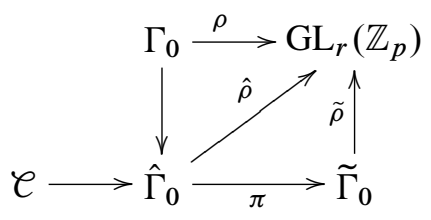

where $\hat{\rho}$ exists due to the universal property of profinite groups. Since any element commuting with $\rho\left(\Gamma_{0}\right)$ would be in the center of its Zariski closure, we see that $\hat{\rho}\left(\hat{\Gamma}_{0}\right)$ also has trivial center. The congruence kernel $\ell=\operatorname{ker} \pi \cap \hat{\Gamma}_{0}$ is central, hence is killed by $\hat{\rho}$. Thus, $\hat{\rho}$ factors through the congruence completion and we obtain $\tilde{\rho}$; all the maps are continuous.

By Proposition 3.3 and the claim above,

$$
\widetilde{\Gamma} \cong \prod_{l} \prod_{\overline{\mathfrak{m}}} \mathrm{SL}_{n}\left(\mathcal{R}_{l, f(\overline{\mathrm{m}})}\right)
$$

where $l$ goes over all primes and $\overline{\mathfrak{m}}$ goes over all the maximal ideals in $\mathbb{F}_{l}[\overline{\boldsymbol{x}}]$. The rings $\mathcal{R}_{l, f(\overline{\mathrm{m}})}$ are local, so each of the local factors $\operatorname{SL}_{n}\left(\mathcal{R}_{l, f(\overline{\mathrm{m}})}\right)$ has the congruence subgroup property. Since $\widetilde{\Gamma}_{0}$ has finite index in $\widetilde{\Gamma}$, we may assume that $\widetilde{\Gamma}_{0}$ is obtained by replacing finitely many of the local factors by a congruence subgroup.

The image of $\tilde{\rho}$ is a pro- $p$ torsion-free group, so for any $l \neq p$ the image of the $l$-adic factors is trivial. We now consider $\tilde{\rho}$ as a representation into $\operatorname{GL}_{r}\left(\overline{\mathbb{Q}}_{p}\right)$. Then $\tilde{\rho}$ may not be irreducible on $\widetilde{\Gamma}_{0}$, but it is completely reducible. Each irreducible component can be written as a tensor product of irreducible representations of finitely many local factors and a 1-dimensional representation of the product of the other factors, by Remark 2.5. Using the fact that $\Gamma$ has finite abelianizations and that it is dense in $\mathrm{SL}_{n}\left(\mathcal{R}_{p, f(\overline{\mathrm{m}})}\right)$, we see that any abelian image must be finite, hence trivial. It follows that we can regard any irreducible component of $\left.\tilde{\rho}\right|_{\Gamma_{0}}$ as a representation of a finite product of principal congruence subgroups $\prod_{j=1}^{t} \mathrm{SL}_{n}^{\prime}\left(\mathcal{R}_{p, f_{j}}\right)$, establishing the proposition. 


\section{Representations of the local factors}

Put $\mathcal{R}=\mathcal{R}_{p, f}$ and let $\mathfrak{m}$ be the maximal ideal of $\mathcal{R}$. In view of 4.2 we shall study irreducible representations of $\mathrm{SL}_{n}^{\prime}(\mathcal{R})$.

Remark 5.1. If $\mathfrak{p} \unlhd \mathcal{R}$ is a prime ideal so that $\mathcal{R} / \mathfrak{p}$ is a f.g. $\mathbb{Z}_{p}$-module, it is integral over $\mathbb{Z}_{p}$ and we have an equality of Krull dimensions $\operatorname{dim} \mathcal{R} / \mathfrak{p}=\operatorname{dim} \mathbb{Z}_{p}=1$. Conversely, if $\operatorname{dim} \mathcal{R} / \mathfrak{p}=1$ then it is finite over $\mathbb{Z}_{p}$ ([6], 6.43). We say that $\mathfrak{p}$ has dimension 1.

Theorem 5.2. Let $v: \mathrm{SL}_{n}^{\prime}(\mathcal{R}) \rightarrow \mathrm{GL}_{d}\left(\overline{\mathbb{Q}}_{p}\right)$ be an irreducible representation. Then there exists a finite index subgroup $\Lambda \leq \mathrm{SL}_{n}^{\prime}(\mathcal{R})$ so that $\left.v\right|_{\Lambda}$ decomposes as a direct sum $\bigoplus v_{i}$, and each component has the form

$$
v_{i}: \Lambda \stackrel{\alpha}{\rightarrow} \prod_{j=1}^{s(i)} \mathrm{SL}_{n}\left(\overline{\mathbb{Q}}_{p}\right) \stackrel{\tau}{\rightarrow} \mathrm{GL}_{d}\left(\overline{\mathbb{Q}}_{p}\right)
$$

where each of the components of $\alpha$ is a specialization and $\tau$ decomposes as a tensor product of irreducible algebraic representations.

For the proof we proceed in several steps. We say that a pro- $p$ group (pro- $p$ ring) is f.g. if it is topologically f.g., i.e., if it has a f.g. dense subgroup (subring).

Proposition 5.3. Let $v: \mathrm{SL}_{n}\left(\mathrm{~m}^{l}\right) \rightarrow \mathrm{GL}_{d}\left(\overline{\mathbb{Q}}_{p}\right)$ be a continuous representation.

(a) There exists an ideal $0 \neq U \unlhd \mathcal{R}$ so that $\operatorname{SL}_{n}(U) \leq \operatorname{ker} v$ and $\mathcal{R} / U$ is a f.g. $\mathbb{Z}_{p}$-module (in particular, $\operatorname{ker} v \neq 1$ ).

(b) If $v$ is irreducible, then $U$ can be taken to be a radical ideal, all of whose associated prime ideals have dimension 1.

Remark 5.4. Bass's stable structure theorem ([2], 4.2, 5.1) asserts that if $\mathcal{A}$ is semilocal and $N \unlhd \mathrm{SL}_{n}(\mathcal{A})$ is any normal subgroup, there exists $0 \neq U \unlhd \mathcal{A}$ so that $\mathrm{SL}_{n}(U) \leq N$. However, here we need this stronger result.

Proof. $\mathrm{SL}_{n}\left(\mathrm{~m}^{l}\right)$ is f.g., since it is (topologically) generated by elementary matrices (Prop. 2.1) and the ideal $\mathrm{m}^{l}$ is f.g. Let $F \subseteq \overline{\mathbb{Q}}_{p}$ be the field generated by the entries of the image of $v$. Then $F$ is a f.g. algebraic extension of $\mathbb{Q}_{p}$, hence finite over $\mathbb{Q}_{p}$. We regard $v$ as a representation $v: \mathrm{SL}_{n}\left(\mathrm{~m}^{l}\right) \rightarrow \mathrm{GL}_{d}(F)$. Let $\mathcal{O}_{p}^{\prime}$ be the ring of integers of $F$. The image of $v$ is compact since $\mathrm{SL}_{n}(\mathcal{R})$ is, therefore it lies in a subgroup conjugate to $\mathrm{GL}_{d}\left(\mathcal{O}_{p}^{\prime}\right)$ ([16], IV.2). This is a pro- $p$ analytic group, hence every closed subgroup is f.g. ([6], 8.1, 3.11).

Fix distinct $i, j \in\{1, \ldots, n\}$. The image under $\rho$ of $E_{i j}\left(\mathrm{~m}^{l}\right)$ is closed, abelian and f.g., hence is isomorphic to $\mathbb{Z}_{p}^{m} \times H$ for some $m \in \mathbb{N}$ and a finite group $H$ ([6], Ex. 1.23). It follows that $S_{i j}=\operatorname{ker} v \cap E_{i j}\left(\mathfrak{m}^{l}\right)$ is not trivial, since $\mathfrak{m}^{l}$ has 
finite index in $\mathcal{R}$ which is not f.g. as an abelian pro- $p$ group. Identify $S_{i j}$ with the corresponding set in $\mathcal{R}$, and let $J_{i j}$ be the ideal generated by $S_{i j}$. Since $n \geq 3$, we may use the commutation relation

$$
\left[E_{i j}(a), E_{j v}(s)\right]=E_{i v}(a s) \quad(i \neq v)
$$

twice to see that if $s \in S_{i j}, a \in \mathfrak{m}^{2 l}$, then $a \cdot s \in S_{u v}$ for any distinct $u, v \in\{1, \ldots, n\}$. Take $U=\mathrm{m}^{2 l} J_{i j}$; then $\mathcal{R} / U$ is a finite $\mathbb{Z}_{p}$-module, and it follows from 2.1 that $\operatorname{SL}_{n}(U)=\operatorname{EL}_{n}(U) \subseteq \operatorname{ker} v$, as needed.

Suppose now that $v$ is irreducible; then the Zariski closure of its image is reductive. We may again pass to a finite index subgroup and assume that the Zariski closure of $v\left(\mathrm{SL}_{n}\left(\mathfrak{m}^{l}\right)\right)$ is connected and semisimple (recall that $\mathrm{SL}_{n}(\mathcal{R})$ has finite abelianizations). Consider the projection $\pi_{U}: \operatorname{SL}_{n}\left(\mathfrak{m}^{l} / U\right) \rightarrow \operatorname{SL}_{n}\left(\mathfrak{m}^{l} / \sqrt{U}\right)$ between principal congruence subgroups in the respective quotient rings; it is indeed onto by 2.2. Since $U$ contains a power of its radical, it is easy to see that ker $\pi_{U}$ is unipotent, hence its image under $v$ is trivial, and we may take $U$ to be a radical ideal. $\mathcal{R}$ is Noetherian, so $U$ is a finite intersection of prime ideals, say $U=\bigcap_{i=1}^{s} \mathfrak{p}_{i}$. The quotients $\mathcal{R} / \mathfrak{p}_{i}$ are also finite over $\mathbb{Z}_{p}$, asserting the last claim.

The following lemmas address the structure of $\mathcal{R} / U$; the first one is a variant of the Chinese remainder theorem (cf. [1], 1.10):

Lemma 5.5. Let $\mathfrak{p}_{1}, \ldots, \mathfrak{p}_{s}$ be distinct prime ideals of dimension 1 in $\mathcal{R}$. Then the natural map

$$
\phi: \mathcal{R} / \bigcap_{i=1}^{s} \mathfrak{p}_{i} \rightarrow \prod_{i=1}^{s} \mathcal{R} / \mathfrak{p}_{i}
$$

is injective and its image contains a finite index ideal.

Proof. Injectivity is clear. We claim that for some $m$, the image of $\mathcal{R} / \cap \mathfrak{p}_{i}$ contains the product $\prod_{i=1}^{s} \mathfrak{m}^{m} / \mathfrak{p}_{i}$. It follows from the dimension assumption and from the fact that for all $j \neq i, \mathfrak{p}_{i}, \mathfrak{p}_{j} \subseteq \mathfrak{m}$ by locality of $\mathcal{R}$, that the only prime ideal containing $\mathfrak{p}_{i}+\mathfrak{p}_{j}$ is the maximal ideal $\mathfrak{m}$, so this is an $\mathfrak{m}$-primary ideal. As any ideal contains a power of its radical, we have $\mathfrak{m}^{t_{i j}} \subseteq \mathfrak{p}_{i}+\mathfrak{p}_{j}$ for some $t_{i j}$. Take $t=\max _{i j}\left(t_{i j}\right)$ and $m=t \cdot(s-1)$. It is enough to show that $\left(y_{1} \ldots y_{s-1}, 0, \ldots, 0\right)$ is in the image of $\phi$ for $y_{1}, \ldots, y_{s-1} \in \mathfrak{m}^{t}$. Write for $i=1, \ldots, s-1: y_{i}=u_{i}+v_{i}$ $\left(u_{i} \in \mathfrak{p}_{1}, v_{i} \in \mathfrak{p}_{i+1}\right)$. It is easy to check that

$$
x=v_{1} \ldots v_{s-1}=\left(y_{1}-u_{1}\right) \ldots\left(y_{s-1}-u_{s-1}\right),
$$

as desired.

Lemma 5.6. Let $\mathfrak{p}$ be a prime ideal of dimension 1 in $\mathcal{R}$. Put $S_{0}=\mathcal{R} / \mathfrak{p}$ and let $S$ be the integral closure of $S_{0}$ in its field of fractions. Then $S$ is a complete discrete valuation ring $(D V R)$ and $S_{0}$ has finite index in $S$ as abelian groups. 
Proof. $S$ is a complete local pro- $p$ domain of dimension 1 by [8], lemma 6 . Since $\delta$ is integrally closed by assumption, it is a complete DVR. As $\delta_{0}$ and $\delta$ have the same field of fractions, their free ranks as $\mathbb{Z}_{p}$-modules are equal and finite by hypothesis. It follows that $\delta_{0} \leq_{f} S$.

The next lemma is essentially part of the proof of the superrigidity theorem ([3], 16.2), which we reproduce here for completeness:

Lemma 5.7. Let $S$ be a pro- $p D V R, K$ its field of fractions, $H=R_{K / \mathbb{Q}_{p}}\left(\mathrm{SL}_{n}\right)$. Let $F$ be a finite extension of $\mathbb{Q}_{p}$ and $\tau: \mathrm{SL}_{n}^{\prime}(\mathcal{S}) \rightarrow \mathrm{GL}_{d}(F)$ a continuous representation. Then $\tau$ coincides on a finite index subgroup with a rational representation $T: H \rightarrow$ $\mathrm{GL}_{d}$ defined over $F$.

Proof. $\mathrm{SL}_{n}(S)=H_{\mathbb{Z}_{p}}$ since $S$ is a DVR (cf. [24], 6.1.3). As before, the image of $\tau$ is contained in a subgroup conjugate to $\mathrm{GL}_{d}\left(\mathcal{O}_{p}^{\prime}\right), \mathcal{O}_{p}^{\prime}$ the ring of integers of $F$, so this is a continuous map between analytic pro- $p$ groups. Hence $\tau$ is analytic ([6], ch. 9); let $\mathscr{L}(\tau)$ be its tangent map at the identity. Since SL is semisimple and simply-connected, so is $H$, and there exists a unique homomorphism of algebraic groups $T: H \rightarrow \mathrm{GL}_{d}$, defined over $F$, whose tangent map is $\mathscr{L}(\tau)$. Therefore, $T$ agrees locally over $F$ with $\tau$, so they coincide on a finite index subgroup.

Proof of 5.2. By 5.3, $v$ factors, on a suitable finite index subgroup, as a representation of $\operatorname{SL}_{n}^{\prime}(\mathcal{R} / U)$, which can be seen by $5.5,5.6$ as a finite index subgroup in $\prod_{j=1}^{s} \mathrm{SL}_{n}\left(\mathcal{S}_{j}\right)$, with $S_{j}$ a pro- $p$ DVR. Such a subgroup can be assumed to be of the form $\prod \mathrm{SL}_{n}^{\prime}\left(\delta_{j}\right)$ since each factor has the CSP. The map $\alpha: \mathrm{SL}_{n}^{\prime}(\mathcal{R}) \rightarrow \prod \mathrm{SL}_{n}\left(\delta_{j}\right)$ is a specialization since it is induced by a ring homomorphism $\mathcal{R} \rightarrow \prod S_{j}$.

Fix an index $j$ and put $S=S_{j}, K, H$ as in 5.7. Let $\left\{\sigma_{1}, \ldots, \sigma_{m}\right\}$ be the distinct embeddings of $K$ in $\overline{\mathbb{Q}}_{p}$. Then $\mathrm{SL}_{n}(\delta)$ embeds in $H_{\overline{\mathbb{Q}}_{p}} \cong \prod_{i=1}^{m} \mathrm{SL}_{n}\left(\overline{\mathbb{Q}}_{p}\right)$, via the map $g \rightarrow\left(g^{\sigma_{1}}, \ldots, g^{\sigma_{m}}\right)$ where the $\sigma_{i}$ act on the matrix $g$ in the obvious way ([19], 12.4). The theorem follows from this remark together with 5.7 and Section 2.5.

Putting everything together, the proof of the main theorem now follows easily:

Proof of 1.4. We begin with the $p$-adic case. Let $\rho: \Gamma \rightarrow \mathrm{SL}_{r}\left(\mathbb{Z}_{p}\right)$ be an irreducible representation. By 4.2, $\rho$ decomposes on a finite index subgroup as a tensor product of continuous irreducible representations $\mathrm{SL}_{n}^{\prime}\left(\mathcal{R}_{p, f}\right) \rightarrow \mathrm{GL}_{r}\left(\overline{\mathbb{Q}}_{p}\right)$. In view of 5.2, each of these representations coincides, again on a finite index subgroup, with a tensor product $\otimes \alpha_{j} \circ \tau_{j}$, where the $\alpha_{j}$ are specializations into $\overline{\mathbb{Q}}_{p}$ and $\tau_{j}$ are rational representations of $\mathrm{SL}_{n}\left(\overline{\mathbb{Q}}_{p}\right)$. Taken together, we see that on a finite index subgroup $\Gamma_{0} \leq_{f} \Gamma, \rho$ decomposes as a direct sum of tensor products of specializations:

$$
\Gamma_{0} \stackrel{\alpha}{\rightarrow} \prod_{j=1}^{t} \mathrm{SL}_{n}\left(\overline{\mathbb{Q}}_{p}\right) \stackrel{\tau}{\rightarrow} \mathrm{GL}_{r}\left(\overline{\mathbb{Q}}_{p}\right)
$$


The complex case now follows by applying the isomorphism $\Phi: \overline{\mathbb{Q}}_{p} \cong \mathbb{C}$ from 4.1 to the specializations $\alpha$ and the representations $\tau$. Finally, the semisimple case follows directly from the irreducible one.

\section{Appendix}

We assert that the group $G=\mathrm{SL}_{n}(\mathbb{C})$ has no irreducible representations of dimension $n^{2}$. [7] serves as a reference for this appendix. Recall that any irreducible representation of $G$ is determined by a unique highest weight, which in turn is defined by an $(n-1)$-tuple of nonnegative integers $\left(a_{1}, \ldots, a_{n-1}\right)$. This representation is denoted by $\Gamma_{a_{1}, \ldots, a_{n-1}}$, and we put for $a_{1}, a_{2}, \ldots, a_{n-1} \geq 1$ : $d\left(a_{1}, \ldots, a_{n-1}\right)=$ $\operatorname{dim} \Gamma_{a_{1}-1, \ldots, a_{n-1}-1}$. We have ([7], 15.17):

$$
d=d\left(a_{1}, \ldots, a_{n-1}\right)=\prod_{1 \leq i<j \leq n} \frac{a_{i}+\cdots+a_{j-1}}{j-i}
$$

The following elementary properties are easy consequences of this formula:

(a) $d$ is monotonous in the $a_{i}$;

(b) $d$ is symmetric, i.e., $d\left(a_{1}, a_{2}, \ldots, a_{n-1}\right)=d\left(a_{n-1}, \ldots, a_{2}, a_{1}\right)$;

(c) If $i<j \leq \frac{n-1}{2}$ and $k \geq 2$, then

$$
d\left(1, \ldots, k_{i}, \ldots, 1, \ldots, 1\right)<d\left(1, \ldots, 1, \ldots, k_{j}, \ldots, 1\right) .
$$

Proposition 6.1. The group $\mathrm{SL}_{n}(\mathbb{C})(n \geq 3)$ has no irreducible representations of dimension $n^{2}$.

Proof. An elementary calculation gives

$$
d(2,1,1, \ldots, 1,2)=n^{2}-1 .
$$

By the properties above we obtain that $d(1, \ldots, k, \ldots, l, \ldots, 1)>n^{2}$ for any $k, l \geq 2$ apart from the option (2). Another elementary calculation gives: $d(k, 1, \ldots, 1)=$ $\left(\begin{array}{c}k+n-2 \\ k-1\end{array}\right)$. For $n \geq 6, k \geq 4$ this is larger than $n^{2}$, and by the properties above we have $d(1, \ldots, k, \ldots, 1)>n^{2}$. The cases $n=3,4,5$ can be checked directly; therefore, we only need to check the cases $k=2,3$.

For $k=2$ note that

$$
d\left(a_{1}, \ldots, a_{n-1}, 1\right)=d\left(a_{1}, \ldots, a_{n-1}\right) \cdot \frac{1}{(n-1) !} \cdot \prod_{i=1}^{n-1}\left(a_{i}+\cdots+a_{n}\right)
$$

It is easily checked that if $\overline{\mathbf{a}}=(1,1, \ldots, 2, \ldots 1)$, where 2 is at the index $l$, then $d(\overline{\mathbf{a}})=\left(\begin{array}{l}n \\ l\end{array}\right) \neq n^{2}$. Finally, for $k=3$ note that $d(3,1, \ldots, 1)=\frac{n(n+1)}{2}$, and using (3) we obtain that $d(1,3, \ldots, 1)>n^{2}$ for any $n \geq 4$. The claim now follows from the properties of $d$. 


\section{References}

[1] M. F. Atiyah and I. G. Macdonald, Introduction to commutative algebra. Addison-Wesley, Reading 1969. Zbl 0175.03601 MR 0242802

[2] H. Bass, K-theory and stable algebra. Inst. Hautes Études Sci. Publ. Math. 22 (1964), 5-60. Zbl 0248.18025 MR 0174604

[3] H. Bass, J. Milnor, and J.-P. Serre, Solution of the congruence subgroup problem for $\mathrm{SL}_{n}(n \geq 3)$ and $\mathrm{Sp}_{2 n}(n \geq 2)$. Inst. Hautes Études Sci. Publ. Math. 33 (1967), 59-137. Zbl 0174.05203 MR 0244257

[4] J. W. S. Cassels, Local fields. London Math. Soc. Stud. Texts 3, Cambridge University Press, Cambridge 1986. Zbl 0595.12006 MR 0861410

[5] I. S. Cohen, On the structure and ideal theory of complete local rings. Trans. Amer. Math. Soc. 59 (1946), 54-106. Zbl 0060.07001 MR 0016094

[6] J. D. Dixon, M. P. F. du Sautoy, A. Mann, and D. Segal, Analytic pro- $p$ groups. Cambridge Stud. Adv. Math. 61, 2nd ed., Cambridge University Press, Cambridge 1999. Zbl 0934.20001 MR 1720368

[7] W. Fulton and J. Harris, Representation theory. Graduate Texts in Math. 129, SpringerVerlag, New York 1991. Zbl 0744.22001 MR 1153249

[8] A. Jaikin-Zapirain, On linearity of finitely generated $R$-analytic groups. Math. Z. 253 (2006), 333-345. Zbl 1097.20026 MR 2218704

[9] M. Kassabov and N. Nikolov, Universal lattices and property tau. Invent. Math. 165 (2006), 209-224. Zbl 1139.19003 MR 2221141

[10] D. A. Kazhdan, Connection of the dual space of a group with the structure of its closed subgroups. Funkcional. Anal. i Priložen. 1 (1967), 71-74; English transl. Funct. Anal. Appl. 1 (1967), 63-65 (1967). Zbl 0168.27602 MR 0209390

[11] N. Koblitz, p-adic numbers, p-adic analysis, and zeta-functions. 2nd ed., Graduate Texts in Math. 58, Springer-Verlag, New York 1984. Zbl 0364.12015 MR 0754003

[12] J. Milnor, Introduction to algebraic K-theory. Ann. of Math. Stud. 72, Princeton University Press, Princeton, N.J., 1971. Zbl 0237.18005 MR 0349811

[13] M. S. Raghunathan, On the congruence subgroup problem. Inst. Hautes Études Sci. Publ. Math. 46 (1976), 107-161. Zbl 0347.20027 MR 0507030

[14] J.-P. Serre, Le problème des groupes de congruence pour $\mathrm{SL}_{2}$, Ann. of Math. (2) 92 (1970), 489-527. Zbl 0239.20063 MR 0272790

[15] J.-P. Serre, Algèbre locale. Multiplicités. 3rd ed., Cours au Collège de France, 1957-1958, Lecture Notes in Math. 11, Springer-Verlag, Berlin 1975. Zbl 0142.28603 MR 0201468

[16] J.-P. Serre, Lie algebras and Lie groups. Lecture Notes in Math. 1500, 2nd ed., SpringerVerlag, Berlin 2006. Zbl 0742.17008 MR 2179691

[17] Y. Shalom, Bounded generation and Kazhdan's property (T). Inst. Hautes Études Sci. Publ. Math. 90 (1999), 145-168. Zbl 0980.22017 MR 1813225

[18] Y. Shalom, The algebraization of Kazhdan's property (T). Proc. Internat. Congr. of Mathematicians, Vol. II, EMS Publ. House, Zürich 2006, 1283-1310. Zbl 1109.22003 MR 2275645 
[19] T. A. Springer, Linear algebraic groups. 2nd ed., Birkhäuser, Boston 1998. Zbl 0927.20024 MR 1642713

[20] A. A. Suslin, The structure of the special linear group over rings of polynomials. Izv. Akad. Nauk SSSR Ser. Mat. 41 (1977), 235-252; English transl. Math.-USSR Izv. 11 (1977), 221-238. Zbl 0378.13002 MR 0472792

[21] L. Vaserstein, Bounded reduction of invertible matrices over polynomial rings by addition operations. Preprint 2007.

[22] A. Weil, Basic number theory. Grundlehren Math. Wiss. 144, Springer-Verlag, Berlin 1967. Zbl 0176.33601 MR

[23] O. Zariski and P. Samuel, Commutative algebra. Vol. II, Springer-Verlag, New York 1975. Zbl 0322.13001 MR 0389876

[24] R. J. Zimmer, Ergodic theory and semisimple groups. Birkhäuser, Boston 1984. Zbl 0571.58015 MR 0776417

Received April 14, 2008; revised December 13, 2008

D. K. Shenfeld, Einstein Institute of Mathematics, The Hebrew University of Jerusalem, Edmond J. Safra Campus, Givat Ram, Jerusalem 91904, Israel

Current address: Department of Mathematics, Princeton University, Fine Hall, Washington Road, Princeton, NJ 08544-1000, U.S.A.

E-mail: shenfeld@math.princeton.edu 